\title{
Ganglioneuroma with leiomyomatosis of the urinary bladder: A rare fumour causing frequent micturition and dysuria
}

\author{
Wang Qi, MM; Min Jie, MM; Zhang Tao, MD; Xie Dongdong, MD; Wang Yi, MD; Ding Demao, MM; Chen Lei, \\ MD; Zou Ci, MM; Ma Jiaxing, MM; Zhang Zhiqiang, MM; Wang Daming, MM; Yu Dexin, MD
}

Department of Urology, The Second Affiliated Hospital of Anhui Medical University, Hefei, China

Cite as: Can Urol Assoc J 2014;8(1-2):e44-7. http://dx.doi.org/10.5489/cuaj.1491

Published online January 14, 2014.

\section{Abstract}

We present the case of a 34-year-old female, complaining of frequent micturition and progressive dysuria. By cystoscopy and computerized tomography, we found that she had multiple submucosal protuberant lesions in the entire bladder. After surgical excision, she has thus far showed no further signs of the disease. The postoperative histopathologic examination revealed a ganglioneuroma and leiomyomatosis. Urinary bladder paraganglioma is an unusual tumour; when it is combined with leiomyomatosis, it is extremely rare. We describe the main clinical presentation, diagnostic procedures, and pathologic features. We also review the English literature.

\section{Introduction}

Ganglioneuromas are benign tumours originating from the neural crest cells in the neuroectodermal; they are composed of well-differentiated ganglion cells, nerve fibres and a large number of Schwann cells. These tumours are uncommon compared with other nervous system tumours. ${ }^{1}$ Ganglioneuromas account for less than $0.5 \%$ of all primary bladder tumours. ${ }^{2}$ They are most typically found in the posterior mediastinum and the retroperitoneal space, and less commonly in the adrenal glands, head and neck, and are extremely rare in the pelvis. Ganglioneuromas may cause urinary tract obstruction with lower urinary tract symptoms, as well as pelvic pain. According to the case reports from the Mayo Clinic, gross hematuria and hypertension are the most common clinical presentations. ${ }^{3}$

Leiomyomas of the urinary bladder are also relatively rare, and constitute less than $0.43 \%$ of all bladder tumours. ${ }^{4}$ Moreover, the incidence of bladder leiomyomasis is much lower. They are benign tumours derived from mesenchymal bladder and are typically asymptomatic. Dysuria and hematuria are possible manifestations of these tumours. ${ }^{5}$

We present a unique case of ganglioneuroma with leiomyomatosis arising from the urinary bladder. The features are characterized and confirmed by pathological studies. In addition, ours is the first reported case of this complex lesion.

\section{Case report}

The patient, a previously healthy 34-year-old female, presented with a 1-year course of frequent micturition, dysuria, without gross hematuria, or pelvic pain. She had no history of tumour or trauma. Two months before presentation, she was experiencing nocturia up to 6 to 7 times per evening. On physical examination, the bladder showed no filling, with only mild tenderness. Laboratory testing revealed a white blood cell count of $16.37 \times 10^{9} / \mathrm{L}$ with $88.1 \%$ neutrophils. Creatinine was $183 \mathrm{uM}$ with a blood urea nitrogen (BUN) of $9.16 \mathrm{mM}$. Urine cytological examination was performed 3 times and was negative. Abdominal pelvic enhanced computed tomography (CT) scan examinations suggested multiple tumours within the bladder. Cystoscopy revealed the appearance of multiple submucosal placeholder uplift in the area of the left wall, top wall and the right side of the trigone, and a newly-formed blister-like appearance on the surface of the top wall. There was obvious compressive deformation of the bilateral ureteral openings. Pathologic report of the biopsy showed mild hyperplasia in the urothelium, and subepithelial tissue edema with chronic inflammatory cell infiltration.

As this was a young, generally healthy patient with high requirements for quality of life and a large tumour burden, neither transurethral resection nor partial cystectomy was pursued. We felt that these procedures could not completely remove the myometrial lesions or relieve the obstructions; moreover, she would likely remain with a high chance of 
recurrence with these therapies. Therefore, a combination of radical cystectomy and sigmoid colon orthotopic neobladder formation was adopted. During the operation, due to the obvious adhesion of the uterus to the abdominal wall and bladder and the bilateral ureter dilation, the uterine ligaments were cut, followed by integral resection of the bladder and uterus with both ovaries preserved. A section of $10-\mathrm{cm}$ long pedicled colon loop was selected and sutured continuously with 2-0 absorbable suture to form the neobladder where the ureters were replanted. Finally, catheter and pelvic drainage tubes were indwelled.

\section{Pathology}

The postoperative anatomical specimens showed diffuse polypoid protuberances ranging from 1.0 to $2.5 \mathrm{~cm}$ in diameter in the bladder, and adhesion of the bladder to the uterus, with indistinct boundaries between the bladder and the uterus (Fig. 1). Microscopy revealed a large number of enlarged ganglions into a nodularformation. Moreover, part of the smooth muscle cells had proliferated, the arrangement of cell beam took a swirl or fence shape, and multiple nodules were formed with the bladder wall and cervix involved (Fig. 2, Fig. 3, Fig. 4). The pathological diagnosis was bladder ganglioneuroma with combined leiomyomatosis. The lesions involved the cervix and no residual tumour was seen on the left or right urethral cutting margin. Also, 4 pieces of the left obturator lymph nodes were negative.

\section{Discussion}

Ganglioneuromas include well-differentiated ganglion cells, axons, satellite cells, a large number of Schwann cells, and fibrous stroma. These tumours rarely occur in the urinary

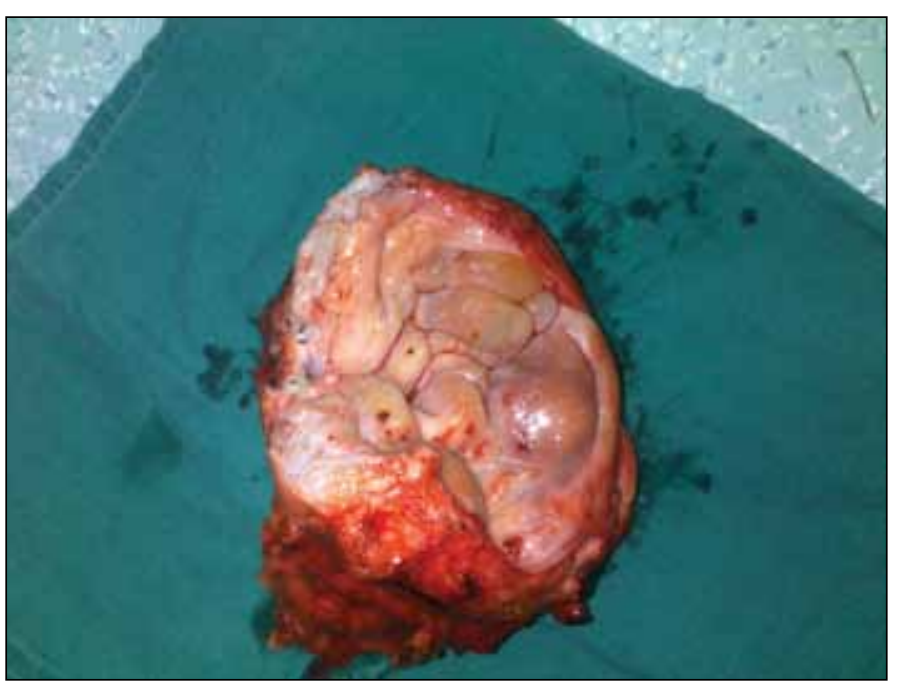

Fig. 1. The postoperative anatomical specimens. bladder. In the early embryonic epiblast, some cells develop into the neural system and thus become neural ectoderm, including the neural tube and neural crest. ${ }^{6,7}$ The neural tube is a structures that forms the brain, spinal cord, and retinal epithelium. The neural crest differentiates into cells, such as ganglion cells, Schwann cells, melanocytes, and adrenal medullary chromaffin. ${ }^{8}$ Ganglioneuroma is generally a unilateral disease; if both sides are involved, a genetic history should be considered. It is mainly associated with autosomal dominant. Ganglioneuroma mostly occur in the posterior mediastinum, followed by retroperitoneal space, adrenals and brain (the third ventricle, temporal lobe and frontal lobe most commonly). They are rare in the spine and its incidence in the pelvis is very low. ${ }^{9,10}$

Bladder leiomyoma is the most common type of nonepithelial bladder neoplasm. Its etiology is not clear, and it has a higher incidence in females in their third to fifth decade of life. ${ }^{11}$ These tumours can be divided into 3 types: (1) submucosal, (2) intramural, and (3) subserosal. They can occur in any part of the urinary bladder. ${ }^{12}$ Bladder leiomyomas show a similar age-adjusted incidence in women as uterine fibroids; these 2 conditions also have similar histologic findings and this similarity suggests that tumour growth is related to hormone levels.

Both bladder ganglioneuromas and leiomyomas have no typical manifestations. However, they share similar symptoms. Both are characterized by gradual growth of a local mass, and accompanied by occasional secretion of chemical neurotransmitters or hormones. Generally there are no obvious symptoms in the early stage. When the tumour volume increases to a certain extent, bladder capacity is decreased. This consequently leads to the emergence of frequent urination urgency symptoms. The close location of the tumour to the bladder neck opening would result

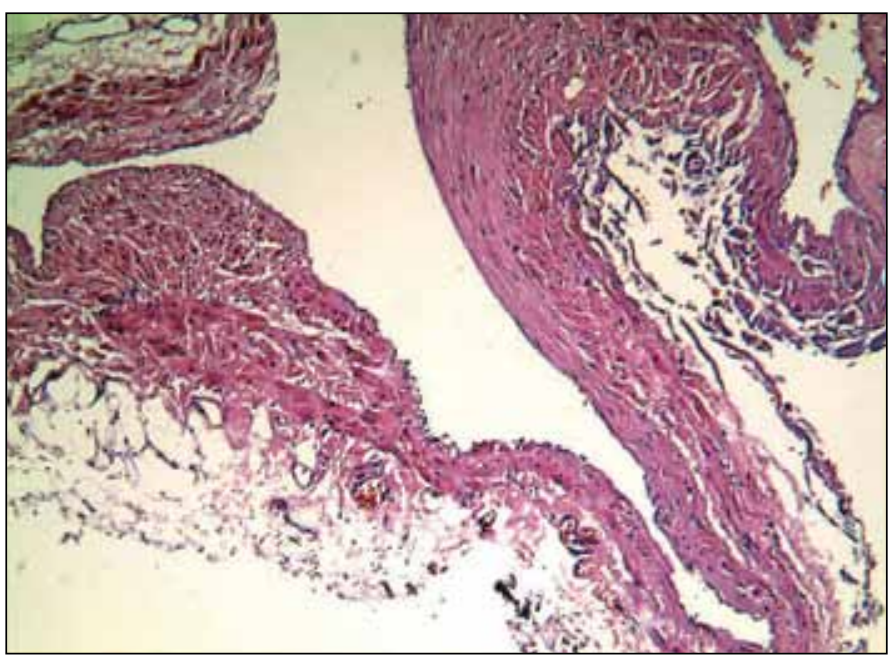

Fig. 2. Histological slides (hematoxylin phloxine saffron stain) at 10x magnification from surgical pathology, demonstrating that the bladder smooth muscle cells had proliferated. 
Qi et al.

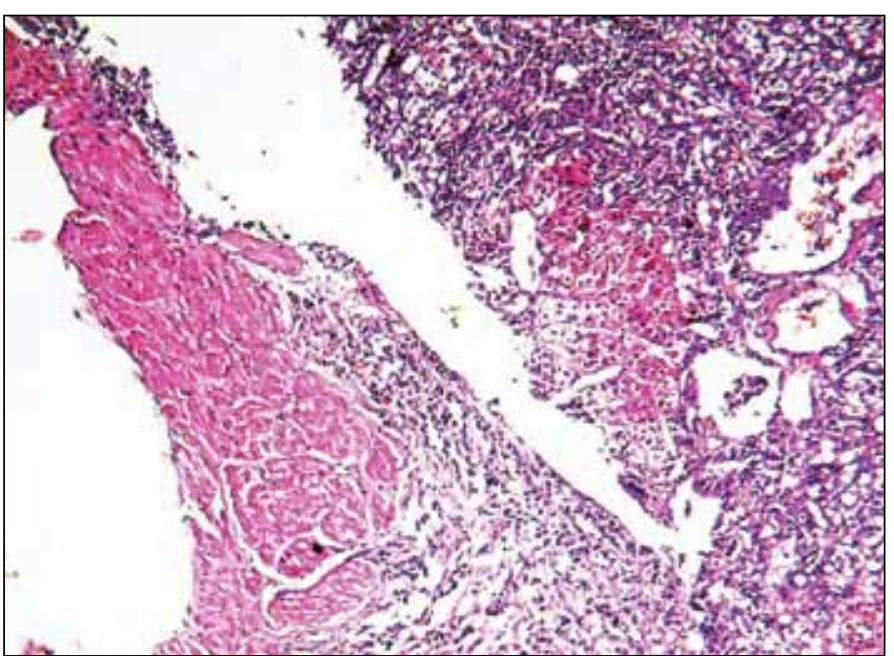

Fig. 3. Histological slides (hematoxylin phloxine saffron stain) at 20x

magnification from surgical pathology, demonstrating that the cell beam had formed multiple nodules.

in dysuria, incomplete bladder emptying, and even urinary retention. If the tumour is located at the opening of the ureters, urine flowing from the ipsilateral upper urinary tract into the bladder would be blocked, which, along with the delay and progression of the disease, would lead to ipsilateral ureter expansion and hydronephrosis. This could further result in impaired renal function and subsequent renal failure. The compression of the adjacent pelvic tissues due to the growth of tumour outside the bladder can cause a series of corresponding symptoms. In females, the involvement of uterus can be characterized by cervical tenderness and irregular vaginal bleeding after physical activity. If there are multiple tumours, the aforementioned symptoms can appear sequentially or simultaneously. ${ }^{13,14}$

The CT findings of bladder ganglioneuromas include round shape, lobulated mass, low density, and uniform appearance. Enhanced CT scan does not show any increases in density. These findings are similar to the CT findings of ganglioneuromas in other more frequently involved regions. ${ }^{15}$ It is worth noting that the appearance of the highly enhanced vascularity with uniform density shadow in this tumour indicates its rich internal arterial blood supply, which is often associated with an increased risk of malignant transformation. ${ }^{16}$ To a certain extent, it confirms the potential transformation from ganglioneuroma into a malignant neuroblastoma. Cystoscopy has the advantage of visualized clarification of location, number, size, and nature of the bladder lesion. Nevertheless, because of the deep extension of the ganglion cells into the bladder smooth muscle layer, distinctive mature ganglion cells may not be found in biopsy specimens, and they are usually interpreted as inflammatory changes. Therefore, cystoscopy can only help to determine tumour morphology and the number of bladder tumours and cannot be regarded as the gold standard for ganglioneuroma diagnosis.

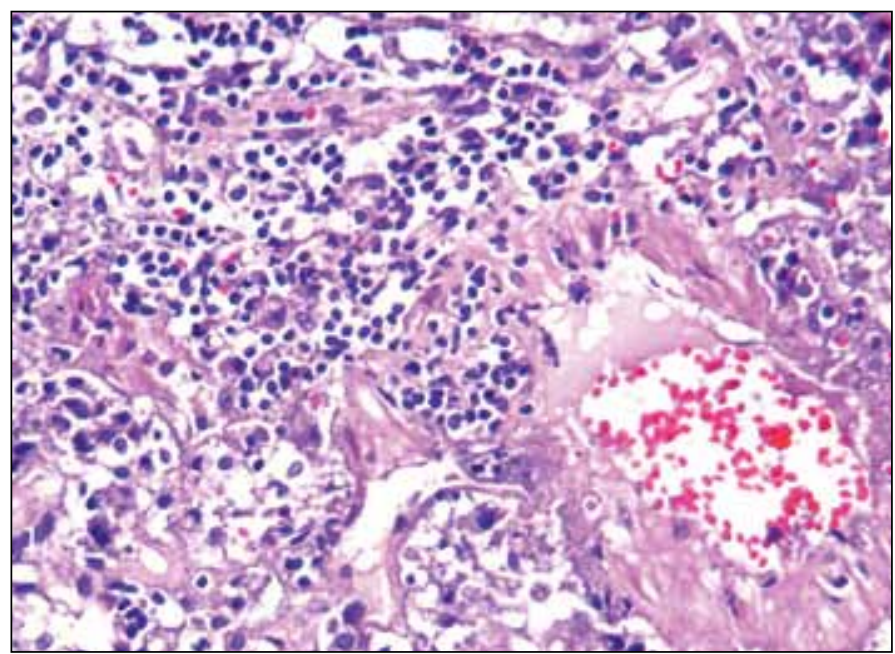

Fig. 4. Histological slides (hematoxylin phloxine saffron stain) at 40x magnification from surgical pathology revealing a ganglioneuroma with combined leiomyomatosis.

As a rare and special bladder tumour, ganglioneuroma should be distinguished from other bladder wall tumours. Bladder cancers are usually multifocal and various in manifestation. When the tumour is relatively large with a wider base and protruding into the bladder, serious testing should be conducted to rule out the possibility of a ganglioneuroma. Benign neoplasm of bladder is rare with leiomyoma as the most common form. Despite their various histopathologic types (e.g., papilloma, inverted papilloma, pheochromocytoma and lymphoma), the benign neoplasms of the bladder are collectively characterized by multiplicity, wide base, circular or semicircular shape, and lumps protruding into bladder. Either paraganglioma or leiomyoma should be differentiated from leiomyosarcoma, which can spread outside of the bladder. In very few female cases, the outside growth of the bladder tumour and its involvement of the organs surrounding the bladder may lead to a misdiagnosis of uterine leiomyoma. The definite diagnosis mainly depends on postoperative pathological examination. ${ }^{17-19}$

Clinically, when it comes to isolated, small volume bladder paragangliomas (which are asymptomatic or without severe symptoms), regular imaging examination and followup cystoscopy instead of surgical therapy are adopted to observe the progress of tumour growth. If the tumour continues to enlarge and leads to decreasing bladder capacity, urine outflow obstruction or renal function impairment, then surgery should be conducted in a timely manner. Unlike transitional cell carcinoma, paragangliomas are mainly located within the bladder wall and have the characteristics of exogenesis, therefore a transurethral resection of the bladder tumour may not be able to remove the tumour completely and may carry the risk of hemorrhage and recurrence. Therefore, the above-mentioned surgical methods are preferred only when the tumour is located in the submucosal with invasion into the whole layer. When there are 
many tumours with severe obstruction and if they involve other organs around the bladder or when partial cystectomy is unable to fully process the lesions, it is recommended to consider radical cystectomy. A radical cystectomy will expand the scope of the resection, if necessary, and will clean the pelvic lymph nodes. At present, radical cystectomy includes both open and laparoscopic surgery. ${ }^{20}$ Compared with traditional open surgery, laparoscopic surgery has the advantages of less blood loss, small trauma, and a shorter postoperative recovery time. Therefore, it has been widely adopted and fully developed in recent years.

\section{Conclusion}

Our case of ganglioneuroma with leiomyomatosis of the urinary bladder presented as frequent micturition and progressive dysuria. When it comes to the diagnosis of spaceoccupying lesions without typical malignant features, a diagnosis of ganglioneuroma with leiomyomatosis of the urinary bladder should be considered. Hopefully, in the near future, the accumulation of long-term follow-up cases can provide more valuable information for better diagnosis and treatment.

Competing interests: Dr. Qi, Dr. Jie, Dr. Tao, Dr. Dongdong, Dr. Yi, Dr. Demao, Dr. Lei, Dr. Ci, Dr. Jiaxing, Dr. Zhiqiang, Dr. Daming and Dr. Dexin all declare no competing financial or personal interests.

This paper has been peer-reviewed.

\section{References}

1. Skovronsky DM, Oberholtzer JC. Pathologic classification of peripheral nerve tumors. Neurosurg Clin $N$ Am 2004;15:157-66. http://dx.doi.org/10.1016/i.nec.2004.02.005
2. Chen $\mathrm{CH}, \mathrm{Boag} \mathrm{AH}, \mathrm{Beiko} \mathrm{DT}$, et al. Composite paraganglioma-ganglioneuroma of the urinary bladder: A rare neoplasm causing hemodynamic crisis at tumor resection. Can Urol Assoc J 2009;3:45-8.

3. Cheng L, Leibovich $B C$, Cheville $J C$, et al. Paraganglioma of the urinary bladder: Can biologic potential be predicted? Cancer 2000;88:844-52. http://dx.doi.org/10.1002/(SICI) 10970142(20000215)88:4<844::AID-CNCR15>3.0.C0;2-1

4. Erdem H, Yildirim U, Tekin A, et al. Leiomyoma of the urinary bladder in asymptomatic women. Urol Ann 2012;4:172-4. http://dx.doi.org/10.4103/0974-7796.102667

5. Ilbey 0, Apaydin E, Gursan A, et al. Bladder leiomyoma: a rare cause of urinary stress incontinence. Arch Ital Urol Androl 2000;72:85-7.

6. Fletcher T, Bradley W. Neuroanatomy of the bladder-urethra. J Urol 1978;119:153-60.

7. Mccaffrey TV, Meyer FB, Michels WV et al. Familial paragangliomas of the head and neck. Arch Otolaryngol Head Neck Surg 1994;120:1211-6. http://dx.doi.org/10.1001/archotol.1994.01880350023005

8. Dahl EV, Waugh IM, Dahlin DC. Gastrointestinal ganglioneuromas. Am J Pathol 1957;33: 953-61.

9. Stout AP. Ganglioneuroma of the sympathetic nervous system. Surg Gynecol Obstet 1947;84:101-10.

10. Uchida K, Kobayashi $S$, Kubota $C$, et al. Michrosurgical excision of ganglioneuroma arising from the C8 nerve root within the neuroforamen. Minim Invas Neurosurg 2007;50:350-54. http://dx.doi. org/10.1055/s-2007-993207

11. Soloway D, Simon MA, Milikowski C, et al. Epithelioid leiomyoma of the bladder: an unusual cause of voiding symptom. Urology 1998;51:1037-9. http://dx.doi.org/10.1016/S0090-4295(98)00016-8

12. Roy MK, Joarder RH, Suruzzaman M, et al. Leiomyoma of the urinary bladder. Mymensingh Med J 2005;14:209-11.

13. Cornella JL, Larson TR, Lee RA, et al. Leiomyoma of the female urethra and bladder: Report of twentythree patients and review of the literature. Am J Obstet Gynecol 1997;176:1278-85. http://dx.doi. org/10.1016/50002-9378(97)70346-6

14. Leesyma JE, Price EB Jr. Paraganglioma of the urinary bladder. Cancer 1971;28:1063-73. http://dx.doi. org/10.1002/1097-0142(1971)28:4<1063::AlD-CNCR2820280433>3.0.C0;2-R

15. Zugor V, Amann K, Schrott KM, et al. Retroperitoneal ganglioneuroma. Aktuelle Urol 2005;36:349-52. http://dx.doi.org/10.1055/s-2004-830278

16. Kimura S, Kawaguchi S, Wada T, et al. Rhabdomyosarcoma arising from a dormant dumbbell ganglioneuroma of the lumbar spine: A case report. Spine 2002;27:E513-7. http://dx.doi.org/10.1097/00007632200212010-00019

17. Zhou M, Epstein Jl, Young RH. Paraganglioma of the urinary bladder: A lesion that may be misdiagnosed as urothelial carcinoma in transurethral resection specimens. Am I Surg Pathol 2004;28:94-100. http:// dx.doi.org/10.1097/00000478-200401000-00011

18. Mouli S, Casalino DD, Nikolaidis P. Imaging features of common and uncommon bladder neoplasms. Radiol Clin North Am 2012;50:301. http://dx.doi.org/10.1016/i.rcl.2012.02.001

19. Usuda H, Emura I. Composite paraganglioma-ganglioneuroma of the urinary bladder. Pathol Int 2005;55:596-601. http://dx.doi.org/10.1111/i.1440-1827.2005.01875.x

20. Jeschke K, Wakonig J, Winzely M, et al. Laparoscopic partial cystectomy for leiomyoma of the bladder wall. J Urol 2002;168:2115-6. http://dx.doi.org/10.1016/S0022-5347(05)64309-1

Correspondence: Dr. Yu Dexin, Chief Physician, Professor, Department of Urology; The Second Affiliated Hospital of Anhui Medical University, Hefei, China; yudx_urology@126.com 